\title{
Unilateral Blinking: Insights from Stereo-EEG and Tractography
}

\author{
Elisabeth Kaufmann ${ }^{1}$ [ $\cdot$ Joanna Bartkiewicz ${ }^{1}$. Nicholas Fearns ${ }^{1} \cdot$ Katharina Ernst $^{1} \cdot$ Christian Vollmar $^{1}$. \\ Soheyl Noachtar ${ }^{1}$
}

Received: 24 January 2021 / Accepted: 2 August 2021 / Published online: 16 August 2021

(c) The Author(s) 2021

\begin{abstract}
To study the neuroanatomical correlate of involuntary unilateral blinking in humans, using the example of patients with focal epilepsy. Patients with drug resistant focal epilepsy undergoing presurgical evaluation with stereotactically implanted EEGelectrodes (sEEG) were recruited from the local epilepsy monitoring unit. Only patients showing ictal unilateral blinking or unilateral blinking elicited by direct electrical stimulation were included $(n=16)$. MRI and CT data were used for visualization of the electrode positions. In two patients, probabilistic tractography with seeding from the respective electrodes was additionally performed. Three main findings were made: (1) involuntary unilateral blinking was associated with activation of the anterior temporal region, (2) tractography showed widespread projections to the ipsilateral frontal, pericentral, occipital, limbic and cerebellar regions and (3) blinking was observed predominantly in female patients with temporal lobe epilepsies. Unilateral blinking was found to be associated with an ipsilateral activation of the anterior temporal region. We suggest that the identified network is not part of the primary blinking control but might have modulating influence on ipsilateral blinking by integrating contextual information.
\end{abstract}

Keywords Eye closure $\cdot$ Epilepsy $\cdot$ DTI $\cdot$ Intracranial EEG

$\begin{array}{ll}\text { Abbreviations } \\ \text { CT } & \text { Computed tomography } \\ \text { DTI } & \text { Diffusion-tensor-imaging } \\ \text { EEG } & \text { Electroencephalography } \\ \text { HARDI } & \text { High-angular resolution diffusion imaging } \\ \text { LPMCd } & \text { Dorsal lateral premotor cortex } \\ \text { LPMCv } & \text { Ventral lateral premotor cortex } \\ \text { MRI } & \text { Magnetic resonance imaging } \\ \text { sEEG } & \text { Stereo-EEG } \\ \text { TLE } & \text { Temporal lobe epilepsy }\end{array}$

Handling Editor: Christoph M Michel.

Christian Vollmar and Soheyl Noachtar are joint senior authors.

Elisabeth Kaufmann

elisabeth.kaufmann@med.uni-muenchen.de

1 Epilepsy Center, Department of Neurology, University of Munich, Marchioninistr. 15, 81377 Munich, Germany

\section{Introduction}

Blinking is essential for facial expression (emotional blinking) and ocular protection (reflective blinking), but can also occur voluntarily, or as conditioned response. Its multifaceted importance demands a detailed understanding of its neuroanatomical control.

Blinking arises mainly by a paroxysmal contraction of the orbicularis oculi muscle, is typically bilateral and of a winkwise character (Ponder and Kennedy 1927). The contraction of the orbicularis oculi muscle is mediated by repetitive electric discharges originating of the facial nerve or its motor nucleus (Holstege et al. 1986; Jacome 1999). Its supranuclear control, however, is still incompletely understood. Indirect information is obtained by lesional or neuroanatomical studies, neuronal tracing, functional neuroimaging studies, as well as trans-/extracranial stimulation experiments. In primates, facial motor function is represented by at least six cortical areas. These include the primary motor (M1), the supplementary motor (M2), the dorsal lateral (LPMCd) and ventral lateral premotor (LPMCv), as well as the rostral (M3) and caudal cingulate (M4) motor cortices (Morecraft et al. 2001; Gong et al. 2005a). Secondary cortical input is attributed to the frontal eye field, the somatosensory cortex, 
the visual cortex, the cerebellum, the superior temporal lobe, as well as limbic structures (Morecraft et al. 2001; Gong et al. 2005a). These wide-spread cortical projections and their cortico-cortical interconnections imply that a complex supranuclear network is involved in the context-related regulation of blinking (Muakkassa and Strick 1979; Morecraft et al. 2004). This hypothesis is further supported by phenomena like the volitional or emotional facial paralysis, i.e. an impaired voluntary activation of facial muscles but normal activation with emotion and vice versa. These phenomena are typically observed after localized brain damage in the anterior opercular region or the midline cortex, the insula, the thalamus, the striatocapsular region or the pons (Foix et al. 1926; Alajouanine and Thurel 1933; Borod et al. 1988; Mao et al. 1989; Hopf et al. 1992, 2000; Bakar et al. 1998; Urban et al. 1998; Holstege 2002).

The gold standard to obtain direct information about the neuroanatomical network of blinking contral in vivo would be electrical cortical stimulation and intracranial electrophysiological recording. Such data, though, is as yet limited for blinking in humans. Here we report on epilepsy patients in whom unilateral blinking was induced by ipsilateral cortical stimulation using stereotactically implanted electroencephalography (EEG) electrodes.

\section{Methods}

This study complies with the ethical guidelines of the University of Munich and was approved by the institutional review board. All patients gave written informed consent to the scientific use of their clinically acquired and anonymized data.

\section{Participants}

The recruitment was started after the first incidental finding of stimulation induced unilateral blinking in 01/2015. Patients with drug resistant focal epilepsy and stimulation induced unilateral blinking were then prospectively recruited from the epilepsy monitoring unit at the University of Munich until 12/2020. Only patients with stereo-EEG (sEEG) electrodes in the temporal and/or frontal lobe and available available documentation of the intracranial stimulation results were considered for the study $(n=60)$.

\section{Video-EEG-Monitoring}

All patients have undergone invasive evaluation with up to twelve stereotactically implanted Spencer depth electrodes (Ad-Tech Medical Instrument Corporation, Racine, USA) with a diameter of $0.86 \mathrm{~mm}$ and $4-14$ contacts, each $5-10 \mathrm{~mm}$ apart and of $5 \mathrm{~mm}$ length. Stereo-EEG was recorded using XLTEK Neuroworks software (Natus Medical Incorporated, San Carlos, USA) and an XLTEK EMU128FS amplifier with a sampling rate of $1000 \mathrm{~Hz}$ and 16 bit A-D conversion. In addition, surface electrodes were placed on the accessible skin surface using the international 10-20 system of electrode placement. Epilepsy syndromes were classified in an interdisciplinary patient management conference based on the available EEG data, seizure semiology, neuropsychological test results, as well as the functional and structural imaging data.

\section{Electrical Intracranial Stimulation}

After successful recording of seizures, electrical stimulation was performed in a referential (monopolar mode) using the same stereotactically implanted depth electrodes. An electrode contact in a non-eloquent and non-epileptic brain area was thereby used as reference. Stimulation was performed on all electrode contacts within as well as in spatial proximity of the planned resection area. Therefore, not all stimulated contacts have been within cerebral gray matter, but also within white matter. Electrical stimulation within white matter might more likely lead to current conduction and thus remote clinical effects. Stimulation parameters were set to biphasic stimulation with a frequency of $50 \mathrm{~Hz}$, a fixed pulse width of $300 \mu \mathrm{s}$, a maximum duration of five seconds, and a stimulation intensity of $1-15 \mathrm{~mA}$. The latter was gradually increased by steps of 1-2 $\mathrm{mA}$ until the maximum stimulation intensity of $15 \mathrm{~mA}$ was reached or any clinical symptoms or side effects occurred. In principle, elicited symptoms have a high localizing value if they occur at low stimulation amplitudes $(<8 \mathrm{~mA})$ and are more unspecific if they occur only at higher stimulation amplitudes. To minimize the risk of seizures, stimulation was only performed with antiseizure medication.

\section{Electrode Localization}

The locations of the intracranial electrodes were precisely determined using co-registered preoperative magnetic resonance imaging (MRI) and postoperative computed tomography (CT) scans. For group visualization, electrode positions were additionally normalized to MNI space, (Montreal Neurological Institute), based on T1 MRI to template registration using SPM software (https://www.fil.ion.ucl.ac.uk/spm/) and mirroring right hemispheric contacts to the left hemisphere. Amira Software (Thermo Fisher Scientific, Waltham, MA, USA) was used for 3-dimensional visualization.

\section{Tractography}

In order to visualize the fiber bundles originating from the stimulation site, probabilistic fiber tracking was 
performed whenever diffusion tensor imaging (DTI) data was available (acquired on a 3 T GE Signa HDx Scanner using 64 diffusion-weighted directions, b-value of $1000 \mathrm{~s} /$ $\mathrm{mm}^{2}, 60$ axial slices with $2.4 \mathrm{~mm}$ slice thickness, $96 \times 96$ in-plane matrix, $220 \mathrm{~mm}$ field of view, TR $16,000 \mathrm{~ms}$, TE $90.2 \mathrm{~ms}$, flip angle $90^{\circ}$, SENSE factor 2 for parallel imaging). The DTI data was resampled to isotropic $1 \mathrm{~mm}$ voxel size and then corrected for eddy current distortions and skull-stripped using the FMRIB Software Library Version 4.1.6 (FSL; http://fsl.fmrib.ox.ac.uk), FSL dtifit was used to create fractional anisotropy (FA) maps. Whole brain tractography was performed using a high-angular resolution diffusion imaging (HARDI) approach (http://trackvis. org/blog/tag/diffusion-toolkit/). Tracking used a 2nd-order Runge Kutta propagation algorithm with an angular threshold of $<35^{\circ}$ between neighboring voxels. The termination criteria of the tracking were either fiber length of more than $200 \mathrm{~mm}$ or reaching a voxel with an FA value $<0.1$. Seeding was implemented using a $5 \mathrm{~mm}$ big sphere around the electrode contact of interest. For visualization, only $10 \%$ of the reconstructed fiber tracks are shown.

\section{Statistical Analysis}

Mean and standard deviation were calculated for quantitative parameters. Relative frequencies are presented as total numbers as well as percentages.

\section{Results}

\section{Stimulation Induced Eyelid Contraction}

Stimulation induced unilateral blinking or eyelid closure was observed in 16/60 (26.7\%) patients (5 men, 11 females; $31.56 \pm 11.04$ years of age; age at disease onset: $18.50 \pm 11.57$ years) The clinical details of the patients are summarized in Table 1.

Ipsilateral eyelid contraction was provoked by stimulation in the anterior temporal lobe, including the temporal pole, uncus, amygdala, hippocampus, parahippocampal and fusiform gyrus (left: $n=6$; right: $n=9$ ) using an amplitude of 1 to $14 \mathrm{~mA}$, as well as in one patient by stimulation in the fronto-orbital region (left: $\mathrm{n}=1$ ) using an amplitude of $4 \mathrm{~mA}$. The latter patient showed a herniation of the frontoorbital cortex into the middle cranial fossa most likely resulting in an anterior temporal stimulation effect. The electrical stimulation of the amygdala/uncus and the anterior part of the parahippocampal/fusiform gyrus were associated with the highest blinking rates, i.e. blinking was observed in 9 out of $47(19.1 \%)$ or 9 out of $48(18.8 \%)$ stimulations, respectively. Lower rates were observed for the anterior hippocampus $(5 / 45 ; 11.1 \%)$ and the posterior fusiform gyrus $(1 / 46 ; 2.2 \%)$. Ipsilateral blinking did not occur upon insular $(\mathrm{n}=49)$, fronto-orbital $(\mathrm{n}=47)$ and parietal stimulations $(n=15)$.

\begin{tabular}{llllll}
\hline ID & Sex & Age & Epilepsy type & MRI & Prior surgery \\
\hline 1 & f & 40 & TLE right & Hippocampal asymmetry right $>$ left & No \\
2 & f & 55 & TLE right & Right hippocampal swelling & No \\
3 & m & 19 & TLE left & Ganglioglioma left hippocampal & $\begin{array}{l}\text { Microresection of gangli- } \\
\text { oglioma left hippocampal }\end{array}$ \\
& & & & & No \\
4 & f & 52 & TLE left & Ependymoma left fronto-temporal & No \\
5 & m & 41 & TLE left & Reduced brain volume left temporal, & \\
& & & & temporal herniation of the frontal lobe, & \\
6 & f & 22 & multifocal & Gliosis left parahippocampal & Resection medulloblastoma \\
7 & f & 37 & TLE left & Schizencephaly & No \\
8 & f & 22 & TLE right & Nonlesional & No \\
9 & f & 31 & Insular left & Nonlesional & No \\
10 & m & 13 & TLE left & Cystic glyotic lesion left temporopolar & Resection plexus carcinoma \\
11 & m & 29 & FTLE right & Nonlesional & No \\
12 & f & 31 & TLE left & HS, cavernoma left frontal & No \\
13 & f & 24 & TLE right & FCD & Anterior temporal resection \\
14 & f & 32 & TLE right & HS & No \\
15 & m & 25 & FLE right & Postcontusional defect right frontal & No \\
16 & f & 32 & FTLE right & HS, FCD right frontal & No \\
\hline
\end{tabular}

$f$ female, FCD focal cortical dysplasia, FLE frontal lobe epilepsy, FTLE frontotemporal lobe epilepsy, $H S$ hippocampal sclerosis, $m$ male, TLE temporal lobe epilepsy 
Low stimulation amplitudes were sufficient to elicit the eyelid contraction if the respective electrode contact was within the gray matter, whereas higher stimulation amplitudes were required when stimulating in white matter regions. The eyelid contraction always began simultaneously with the stimulation onset $(n=16)$ and stopped isochronal with stimulation cessation $(n=15)$. In one patient (ID1), electrical stimulation with $3 \mathrm{~mA}$ in the right parahippocampal gyrus elicited unilateral blinking, which evolved into an epileptic seizure three times. The respective electrode contacts were located in close vicinity to the seizure onset zone. In five patients, post-stimulation after-discharges were elicited at higher stimulation amplitudes $(>9 \mathrm{~mA})$ but blinking always ceased at the end of the stimulation and did not continue during the post-stimulation discharges. A grouped visualization of the identified fronto-temporal SEEG contacts of 8 representative patients is shown in Fig. 1.
Seven patients had a predominant clonic eyelid contraction during temporal stimulation. The stimulation site was in close vicinity to their epileptic zone or even elicited a habitual seizure. The other nine patients, in contrast, showed a tonic eyelid contraction. In some of these patients we observed that lower stimulation amplitudes caused palpebral flutter of the eyelid before tonic contraction at higher stimulation amplitudes. The eyelid on the contralateral side, though, always remained unaffected.

In contrast, the other 44 patients with SEEG electrodes in the fronto-orbital and/or temporal region who did not reveal unilateral blinking upon stimulation did not significantly differ from the study cohort with regard to age, disease duration and epileptic hemispheres. However, they encompassed significantly less females (39\% vs. $75 \%$; $p=0.0190)$ and less patients with temporal lobe epilepsy (34\% vs.68\% $\mathrm{p}=0.0212$ ).
Fig. 1 Grouped visualization of contacts associated with unilateral blinking. The figure summarizes the anatomical location of the SEEG electrode contacts (red dots) that were associated with unilateral blinking or tonic eye closure upon their electrical stimulation in 8 representative patients. The size of the red dots represents the specificity of the observed effect, i.e. small dots representing electrodes where blinking was elicited only at higher stimulation amplitudes $>12 \mathrm{~mA}$ and the biggest dots representing the most specific effects that occurred already at $<4 \mathrm{~mA}$. The individual contact locations were determined via co-registration of the postop CT and preoperative MRI scans and transferred to a common space (MNI space). Contacts located within the right hemisphere were mirrored to the left hemisphere. A Sagittal cut with hippocampal angulation. B Coronal cut through the anterior temporal lobe. The dashed lines in the uppermost figure represent the respective section planes (Color figure online)

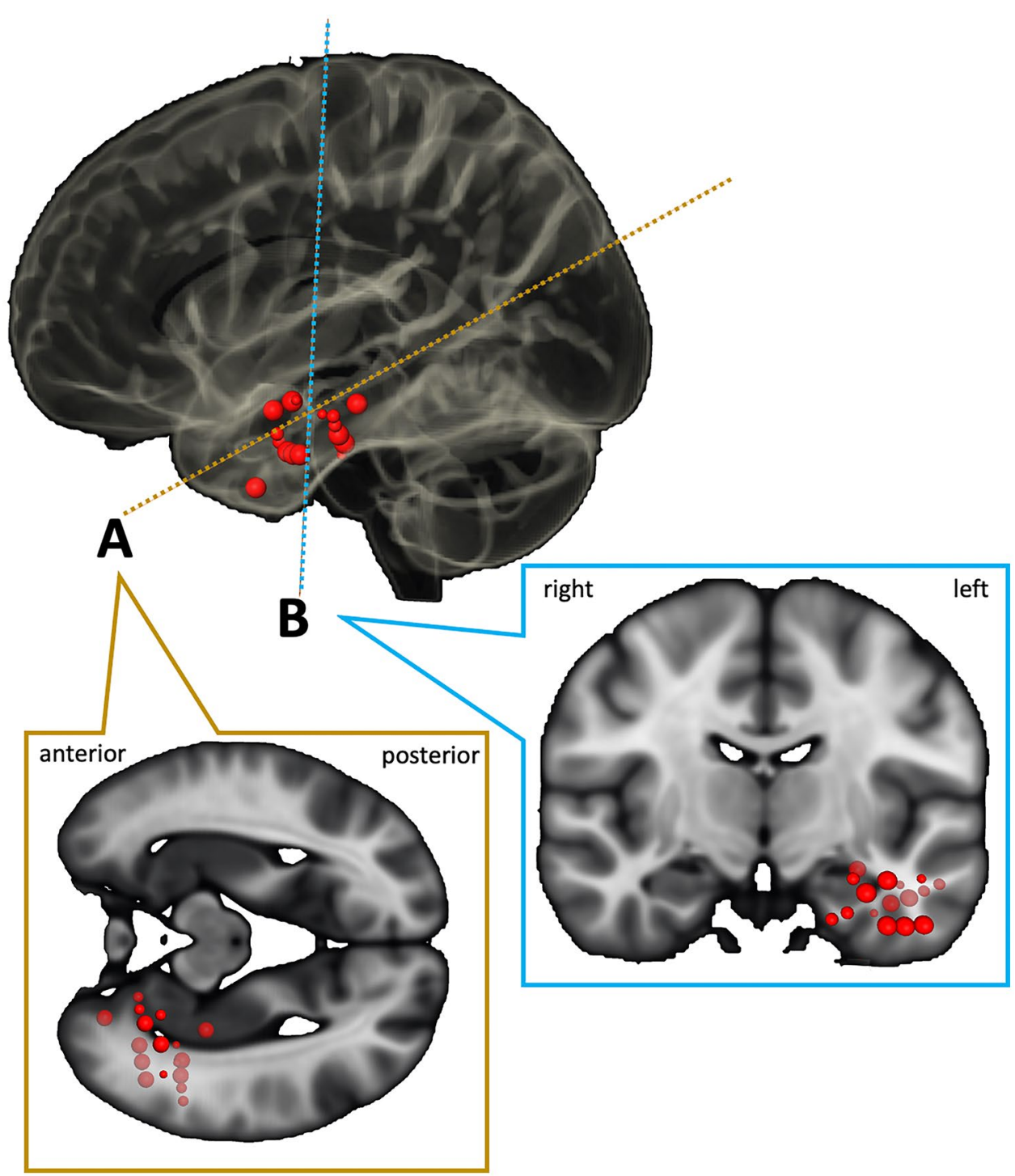




\section{Tractography}

Tractography was performed for two representative patients (ID 3; ID 5) with stimulation induced blinking. Seeding from the left anterior temporal region, where blinking was provoked by electrical stimulation in one patient (ID 3), revealed projections to the ipsilateral cerebellum, fornix and posterior temporal region besides the projections to the frontopolar and pericentral region. In the second patient (ID 5), blinking was induced by electrical stimulation in the herniated left basal fronto-orbital region, where tractography revealed connections not only to the ipsi- but also the contralateral mesial frontopolar region (Fig. 2).

\section{Discussion}

\section{Cerebral Regions Associated with Unilateral Blinking}

Our findings indicate an association of unilateral blinking/eyelid closure with activation of the ipsilateral anterior temporal region. Most frequently, unilateral blinking/eyelid closure was elicited upon electrical stimulation within the amygdala/uncus and fusiform/parahippocampal gyrus. This observation is in line with former reports on fronto-temporal seizure patterns in scalp-EEG recordings during ictal ipsilateral blinking (Benbadis et al. 1996; Henkel et al. 1999; Pestana and Gupta 2007; Kalss et al. 2013), as well as a parahippocampal activation during spontaneous blinking in functional MRTI (fMRI) investigations (Yoon et al. 2005). Of note, in monkeys and rats, retrograde neuronal tracing from the orbicularis oculi muscle revealed higher-order nerval connections spreading into the ipsilateral temporal

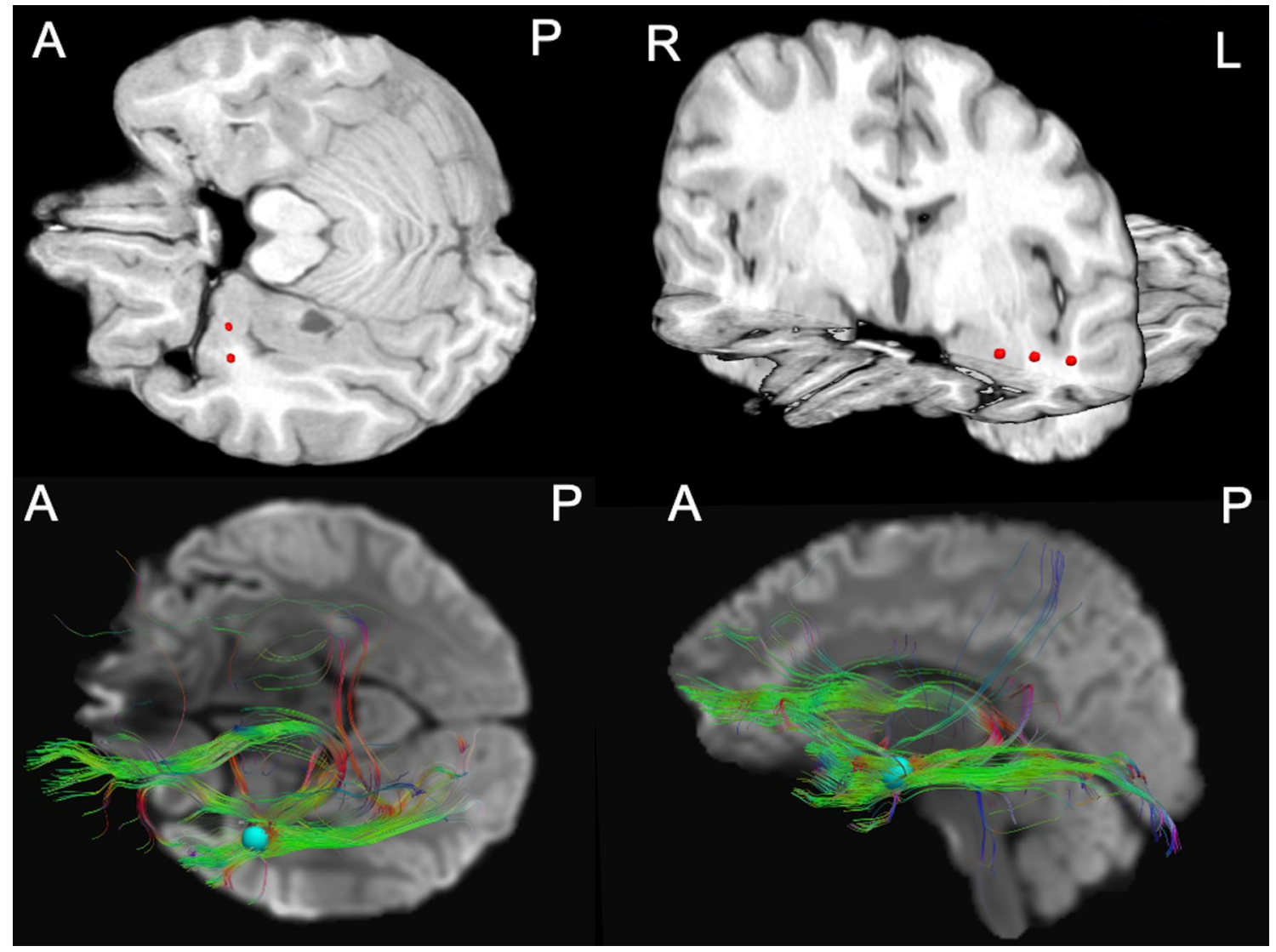

Fig. 2 Tractography results. The upper row shows the location of the electrodes which were associated with unilateral blinking in one representative patient (ID3). The location of the stereo-EEG electrodes was determined by a co-registration of the preoperative MRI and postoperative CT scan.The lower row shows the HARDI fiber track- ing results seeding from a $5 \mathrm{~mm}$ big sphere. The spheres were positioned around one of the electrodes shown in the 2D images above. For reasons of clearness, only $10 \%$ of the reconstructed fibers are shown. As background, average diffusion weighted imaging maps were used. $A$ anterior, $L$ left, $P$ posterior, $R$ right 
lobe, i.e. the superior temporal sulcus or the temporal cortex (Morcuende et al. 2002; Gong et al. 2005a).

\section{Significance of Anterior Temporal Input to the Generation of Blinking}

Presumably, the identified anterior temporal regions have no direct projections to the facial nucleus. It is more likely that they are part of a network of motor cortical projections that influences the primary motor pathway. Previous data suggests a context-dependent activation of this motor network, but the exact way of interaction of the different cortical components or its modulation are still not fully understood (Dimitrova et al. 2002; Yoon et al. 2005).

In our cohort, blinking was elicited most frequently by stimulation of the uncus/amygdala, as well as the anterior fusiform and parahippocampal gyrus. The classical functional attribution to the fusiform gyrus is high level visual processing. In detail, previous electrical stimulation studies delineated circumscribed fusiform regions that are associated with face perception and perception of visual scenes (Parvizi et al. 2012; Mégevand et al. 2014). The parahippocampal gyrus, on the contrary, encodes for emotion processing, visuo-spatial navigation, and episodic memory (Kanwisher et al. 1997; Levy et al. 2001; Kirwan and Stark 2004; Smith et al. 2004; Mitterschiffthaler et al.
2007; Tendolkar et al. 2008). The parahippocampus seems to play an important role in encoding and retrieving contextual associations from a broad cerebral network (Bar et al. 2008; Aminoff et al. 2008, 2013; Peters et al. 2009; NormanHaignere et al. 2012). This is substantiated by our tractography results, showing connections from parahippocampal regions to the limbic, frontal, pericentral, occipital, and cerebellar brain areas. Thus, one might speculate that the mesial temporal regions do not generate blinking, but modulate the frontal corticobulbar projections, while integrating emotional, visuospatial and sensorial input from the cortical and subcortical regions mentioned above (Fig. 3). In other words, also in humans the mesial temporal region probably adjusts the ipsilateral blinking based on its integrated contextual associations (Muakkassa and Strick 1979; Morecraft et al. 2004). Considering the isolated activation of a single muscle during ipsilateral blinking, an alternative conceivable hypothesis could be that the stimulated regions connect to a highly localized but elusive cortical area for ipsilateral blinking that connects with the ipsilateral primary motor cortex and facial nucleus via the ipsilateral cortico-spinal tract.

Ictal blinking has been described in epilepsy patients, most frequently with parieto-occipital and insulo-opercular seizure onsets and typically manifested bilaterally (Marchi et al. 2016; Lagarde et al. 2020; Peltola et al. 2020; Wang
Fig. 3 Hypothesized supranuclear facial motor network. The identified anterior temporal region (greenish) are supposed to integrate information from different secondary facial motor cortices (reddish). By integrating the emotional, visuospatial, and sensorial data, the anterior temporal structures might be able to modulate the primary facial motor projections in a context-dependent manner. In other words, the anterior temporal region might reinforce or inhibit the ipsilateral blinking response depending on e.g. the emotional state or facial sensations like trigeminal pain. An MNI surface template from the commercially available software package Lead DBS was used for the visualization (Horn et al. 2019) (Color figure online)

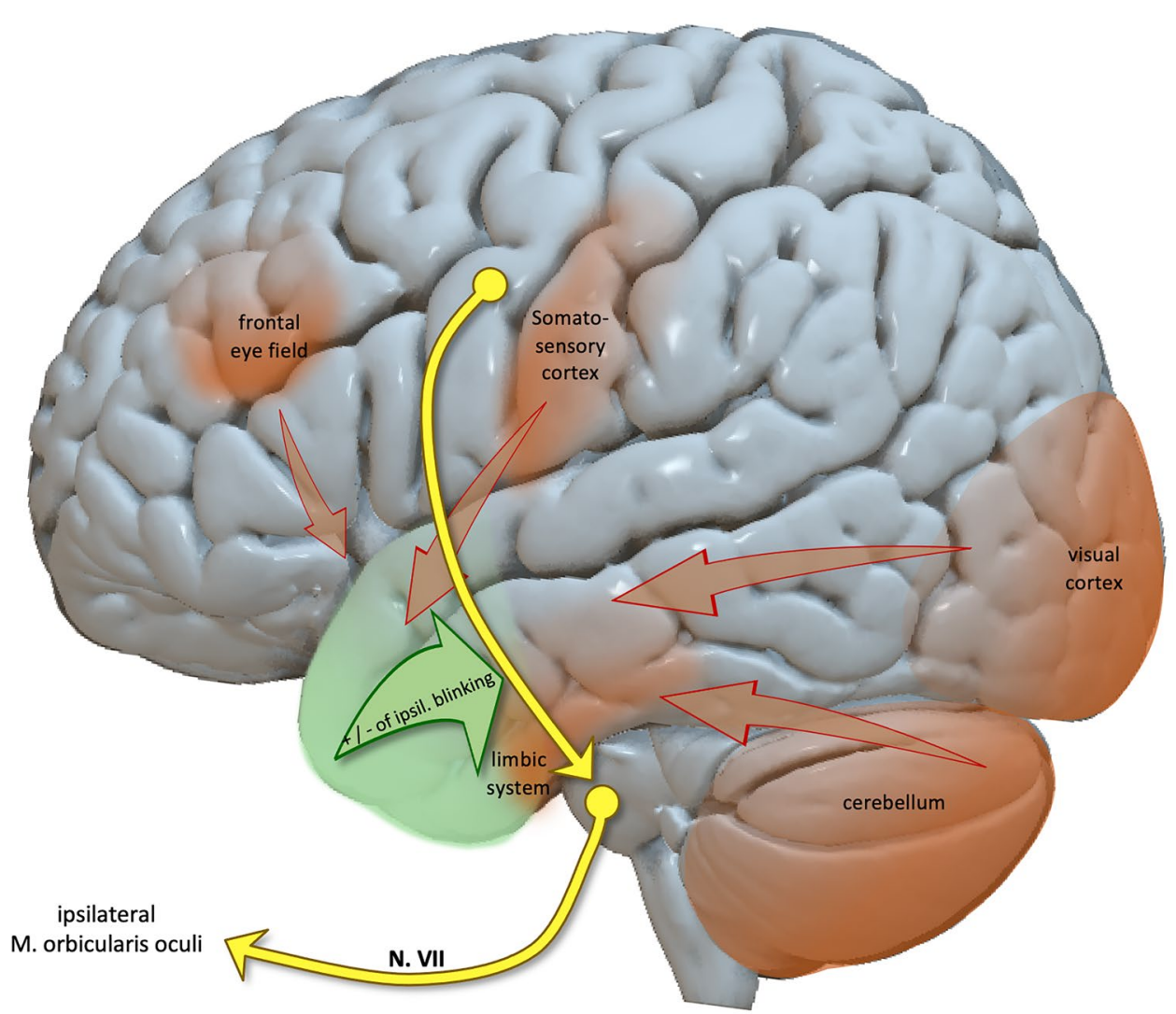


et al. 2020). Unilateral blinking only occurred in association with an activation of the mesial temporal lobe in a large-scaled SEEG study (Lagarde et al. 2020). Similarly, Benbadis et al. observed unilateral blinking during the first $37 \mathrm{~s}$ after the clinical onset of frontal or temporal lobe seizures (Benbadis et al. 1996), which most likely reflected the direct temporal activation or spread of the seizure activity towards the temporal lobe. Further, ictal blinking occurred only after a longer delay in occipital seizures compared to occipito-temporal seizures (Lagarde et al. 2020), potentially due to the longer propagation time towards the temporal lobe or precentral region. This is in line with our finding of an anterior temporal association of unilateral blinking as well as early stimulation experiments describing blinking following amygdaloid or mesial temporal stimulation (Lesser et al. 1985; Benbadis et al. 1996).

\section{Characteristics of Stimulated and Ictal Blinking}

Electrical cortical stimulation induced a palpebral fluttering, which was tonic at higher stimulation amplitudes. Considering that the standard frequency of $50 \mathrm{~Hz}$ was used for electrical stimulation, the flutter/tonic eyelid contraction could be the clinical correlate of a $\sim 50 \mathrm{~Hz}$ blinking, i.e. a highfrequency muscle contraction. This is in line with nerval stimulation studies showing a higher probability of a tonic motor response at higher stimulus frequencies (Begum et al. 2006; Raslan et al. 2017). Similarly, Maillard et al. reported different clinical motor responses in association with frequency changes of the EEG seizure pattern as well as during electrical intracranial stimulation with $1 \mathrm{~Hz}$ versus $50 \mathrm{~Hz}$ (Maillard et al. 2014). In two of our patients clonic unilateral blinking was observed after anterior temmporal stimulation close to the epileptogenic zone which triggered epileptic discharges. These epileptic discharges could also have elicited a clonic motor response; however, we also observed clonic blinking in three patients without after-discharges.

\section{Neuroanatomical Considerations}

Most previous reports of blinking responses suggest an activation of cranial nerves as explanation for ictal or stimulated unilateral blinking. In case of stimulation with subdural electrodes, the facial motor response was thought to be caused by a direct activation of peripheral branches of the motor division of the facial nerve (Begum et al. 2006). Similarly, an ephaptic activation of dural branches of the trigeminal nerve by the fronto-temporal seizure activity was suggested (Sindou et al. 1994; Pestana and Gupta 2007; Jadhav et al. 2016). The so-called trigeminal-facial reflex, also known as blink reflex, might then lead to an ipsilateral face twitching (Livingston and Phillips 1957; Sindou et al. 1994; Gong et al. 2005b; Begum et al. 2006). However, in our patients, a direct activation of cranial nerves seems unlikely (1) because 3D reconstructions of the electrode positions showed clear parenchymal locations. (2) Further, altered sensation in the face would be expected in N. V activation, but was not observed in our patients. Similarly, Bartholow elicited unilateral blinking in one case by intracranial stimulation of the ipsilateral parieto-occipital region (Bartholow 1874), i.e. clearly outside of the primary motor region and distant from the meninges. In our patients, we thus suppose that blinking was facilitated via an activation of the supranuclear facial motor network rather than direct activation of cranial nerves, but this remains speculative. Future studies using low-frequency stimulation with direct measurement of the stimulus-response latency could provide further evidence but were not performed during the clinical evaluation.

Of note, the 16 patients with unilateral blinking encompassed significantly more female patients and more patients with temporal lobe epilepsy compared to the control group without blinking. The latter might be due to a selection bias, as more temporal SEEG electrodes were implanted and available for stimulation in patients with a supposed temporal epileptic zone. Nevertheless, it might also reflect known gender differences in emotion perception and processing including facial expression (Schulte-Rüther et al. 2008; Houstis and Kiliaridis 2009; Mcduff et al. 2017). Interestingly, previous functional MRI studies described an activation of a prefrontal-temporal-parietal network in the context of emotional perspective taking, with a stronger activation of the inferior frontal and superior temporal regions in women compared to men (Schulte-Rüther et al. 2008). To investigate this further, larger-scaled follow-up studies are needed.

\section{Limitations}

Our study is limited by the fact that all our observations were made in patients with epilepsy whose brains might be functionally and structurally reorganized, especially in patients with extensive lesions and in case epileptic seizures or the underlying pathology started early in life. The mean age at disease onset was $18.50 \pm 11.57$ years of age in the cohort of 16 patients with unilateral blinking. However, in the 44 controls it was even slightly earlier $(14.50 \pm 7.97$ years of age) and disease duration did not relevantly differ $(13.06 \pm 7.81$ vs. $14.68 \pm 12.69$ in the control group). Thus, stimulation results obtained in epilepsy patients cannot be generalized without reservation. However, fundamental functions like blinking are typically not affect by this kind of reorganization. Like all evaluations with depth electrodes, our study's findings are potentially confounded by propagation away from the site of electrical stimulation. This is, in particular, relevant in case of stimulation of electrode contacts within the cerebral white matter. 
The induced pathophysiological effects of the latter are not fully understood, but it is likely that the electrical current is conducted ortho- or retrogonally to remote (grey matter) areas which are causal to the observed clinical effects. In other words, the anatomical correlate of ipsilateral blinking in case of blinking induced by stimulation of cerebral white matter is not exactly at the electrode location but within the connectivity of the fibers. Our approach is further limited by the moderate spatial resolution. Driven by the clinical hypothesis and safety aspects, only as few as possible electrodes have been implanted, typically limited to one, i.e. the epileptic hemisphere. This entails that during ictal unilateral blinking the seizure pattern could have had already spread to the contralateral subcortical brain regions. In the simultaneous surface EEG-recording, though, no contralateral seizure pattern was recorded. Moreover, we could not evaluate the presumably important role of the occipital cortex, the basal ganglia and the reticular formation in unilateral blinking (Ebert et al. 1996; Smit 2009; Lagarde et al. 2020), as we did not perform sEEG recordings/stimulation of these regions. Bihemispheric sEEG evaluations of healthy human brains with standardized electrode locations would be of course scientifically desirable but are ethically not acceptable.

Although unlikely, a trigeminal input to the ipsilateral blinking has to be discussed. Neurographic measurements on the N. V or the N. VII were not performed. Thus, more patients have to be examined to consolidate our data and additional neurographic measurement and bilateral intracranial EEG-recording would be desirable.

\section{Conclusion}

We hypothesize that unilateral blinking is associated with an ipsilateral anterior temporal activation. The identified regions, especially the parahippocampal and anterior fusiform gyrus, might have a modulating influence on ipsilateral blinking by integrating contextual information. This is the first study to present intracranial stimulation data on unilateral blinking combined with tractography results.

Acknowledgements The authors are indebted to the patients for their participation in this study, as well as to Renate Piccinotti, Renate Tschakert, Oliver Klein, Elke Scherbaum and Julia Kleve for technical assistance in the EEG-Video-Monitoring unit of the Epilepsy Center, Department of Neurology, University of Munich. Further, we want to thank Prof. Kreth for carrying out the stereotactic implantation of the intracranial EEG-electrodes.

Authors' Contributions EK was responsible for the patient recruitment, data analysis, and drafting the manuscript. JB and CV performed the DTI analyses and critically revised the manuscript. NF performed the group visualization. KE contributed to the retrospective data analysis and critically revised the manuscript. SN supervised the findings of the project, provided critical feedback and helped shape the analysis and manuscript.

Funding Open Access funding enabled and organized by Projekt DEAL. Elisabeth Kaufmann was supported by a fellowship from the German Society for Clinical Neurophysiology and Functional Imaging (DGKN) as well as the Bavarian Gender Equality Grant (BGF) 2017.

\section{Declarations}

Conflict of interest None of the authors has relevant conflicts of interest to report.

Consent to participate All patients gave written informed consent to the scientific use of their clinically acquired and anonymized data.

Ethical Approval All procedures involving human participants were in accordance with the ethical standards of the institutional and/or national research committee and with the 1964 Helsinki declaration and its later amendments or comparable ethical standards.

Informed consent Informed consent was obtained from all individual participants included in the study.

Consent for publication All authors have approved the manuscript and agree with the submission to Brain Imaging \& Behavior.

Data Availability The data that support the findings of this study are available from the corresponding author upon reasonable request.

Code Availability Not applicable.

Open Access This article is licensed under a Creative Commons Attribution 4.0 International License, which permits use, sharing, adaptation, distribution and reproduction in any medium or format, as long as you give appropriate credit to the original author(s) and the source, provide a link to the Creative Commons licence, and indicate if changes were made. The images or other third party material in this article are included in the article's Creative Commons licence, unless indicated otherwise in a credit line to the material. If material is not included in the article's Creative Commons licence and your intended use is not permitted by statutory regulation or exceeds the permitted use, you will need to obtain permission directly from the copyright holder. To view a copy of this licence, visit http://creativecommons.org/licenses/by/4.0/.

\section{References}

Alajouanine T, Thurel R (1933) La diplégie faciale cérébrale forme corticale de la paralysie pseudo-bulbaire (contribution à l'étude de la dissociation des activités volontaire et réflexe). Rev Neurol 40:441-458

Aminoff EM, Schacter DL, Bar M (2008) The cortical underpinnings of context-based memory distortion. J Cogn Neurosci 20:22262237. https://doi.org/10.1162/jocn.2008.20156

Aminoff EM, Kveraga K, Bar M (2013) The role of the parahippocampal cortex in cognition. Trends Cogn Sci 17:379-390. https://doi. org/10.1016/j.tics.2013.06.009

Bakar M, Kirshner HS, Niaz F (1998) The opercular-subopercular syndrome: four cases with review of the literature. Behav Neurol 11:97-103 
Bar M, Aminoff E, Schacter DL (2008) Scenes unseen: the parahippocampal cortex intrinsically subserves contextual associations, not scenes or places per Se. J Neurosci 28(34):8539-8539

Bartholow R (1874) Experimental investigations into the functions of the human brain. Am J Med Sci 66:305-313. https://doi.org/10. 1097/00000441-187404000-00001

Begum T, Ikeda A, Matsuhashi M et al (2006) Ipsilateral facial sensory and motor responses to basal fronto-temporal cortical stimulation: evidence suggesting direct activation of cranial nerves. Epilepsy Res 71:216-222. https://doi.org/10.1016/j.eplepsyres.2006.06.019

Benbadis SR, Kotagal P, Klem GH (1996) Unilateral blinking: a lateralizing sign in partial seizures. Neurology 46:45-48

Borod JC, Koff E, Lorch MP et al (1988) Emotional and non-emotional facial behaviour in patients with unilateral brain damage. J Neurol Neurosurg Psychiatry 51:826-832. https://doi.org/10.1136/jnnp. 51.6 .826

Dimitrova A, Weber J, Maschke M et al (2002) Eyeblink-related areas in human cerebellum as shown by fMRI. Hum Brain Mapp 17:100-115. https://doi.org/10.1002/hbm.10056

Ebert D, Albert R, Hammon G et al (1996) Eye-blink rates and depression. Is the antidepressant effect of sleep deprivation mediated by the dopamine system? Neuropsychopharmacology 15:332-339. https://doi.org/10.1016/0893-133X(95)00237-8

Foix C, Chavany J, Marie J (1926) Diplègie faciolinguomasticatrice d'origine cortico souscorticale sans paralysie des membres. Rev Neurol 33:214-219

Gong S, DeCuypere M, Zhao Y, LeDoux MS (2005a) Cerebral cortical control of orbicularis oculi motoneurons. Brain Res 1047:177193. https://doi.org/10.1016/j.brainres.2005.04.045

Henkel A, Winkler PA, Noachtar S (1999) Ipsilateral blinking: a rare lateralizing seizure phenomenon in temporal lobe epilepsy. Epileptic Disord 1:195-197

Holstege G (2002) Emotional innervation of facial musculature. Mov Disord. https://doi.org/10.1002/mds.10050

Holstege G, Van Ham JJ, Tan J (1986) Afferent projections to the orbicularis oculi motoneuronal cell group. An autoradiographical tracing study in the cat. Brain Res 374:306-320. https://doi. org/10.1016/0006-8993(86)90425-7

Hopf HC, Müller-Forell W, Hopf NJ (1992) Localization of emotional and volitional facial paresis. Neurology 42:1918-1923

Hopf HC, Fitzek C, Marx J et al (2000) Emotional facial paresis of pontine origin. Neurology 54:1217

Horn A, Li N, Dembek TA et al (2019) Lead-DBS v2: Toward a comprehensive pipeline for deep brain stimulation imaging. NeuroImage 184:293-316. https://doi.org/10.1016/j.neuroimage.2018. 08.068

Houstis O, Kiliaridis S (2009) Gender and age differences in facial expressions. Eur J Orthod 31:459-466. https://doi.org/10.1093/ ejo/cjp019

Jacome DE (1999) Monocular involuntary eyelid closure. Acta Neurol Scand 99:130-133

Jadhav T, Bailey C, Maixner W, Harvey AS (2016) Ictal unilateral blinking is an unreliable lateralizing sign in tuberous sclerosis complex. Epilepsy Res 125:58-61. https://doi.org/10.1016/j.eplep syres.2016.06.006

Kalss G, Leitinger M, Dobesberger J et al (2013) Ictal unilateral eye blinking and contralateral blink inhibition-A video-EEG study and review of the literature. Epilepsy Behav Case Reports 1:161165. https://doi.org/10.1016/j.ebcr.2013.10.001

Kanwisher N, McDermott J, Chun MM (1997) The fusiform face area: a module in human extrastriate cortex specialized for face perception. J Neurosci 17:4302-4311

Kirwan CB, Stark CEL (2004) Medial temporal lobe activation during encoding and retrieval of novel face-name pairs. Hippocampus 14:919-930. https://doi.org/10.1002/hipo.20014
Lagarde S, Dirani M, Trebuchon A et al (2020) Ictal blinking in focal seizures: Insights from SEEG recordings. Seizure 81:21-28. https://doi.org/10.1016/j.seizure.2020.07.010

Lesser RP, Lüders H, Klem G et al (1985) Ipsilateral trigeminal sensory responses to cortical stimulation by subdural electrodes. Neurology 35:1760-1763. https://doi.org/10.1212/wnl.35.12.1760

Levy I, Hasson U, Avidan G et al (2001) Center-periphery organization of human object areas. Nat Neurosci 4:533-539. https://doi.org/ $10.1038 / 87490$

Livingston A, Phillips CG (1957) Maps and thresholds for the sensorimotor cortex of the cat. Q J Exp Physiol Cogn Med Sci 42:190 205. https://doi.org/10.1113/expphysiol.1957.sp001250

Maillard L, Gavaret M, Régis J et al (2014) Fast epileptic discharges associated with ictal negative motor phenomena. Clin Neurophysiol 125:2344-2348. https://doi.org/10.1016/j.clinph.2014.03.023

Mao CC, Coull BM, Golper LA, Rau MT (1989) Anterior operculum syndrome. Neurology 39:1169-1172

Marchi A, Bonini F, Lagarde S et al (2016) Occipital and occipital "plus" epilepsies: A study of involved epileptogenic networks through SEEG quantification. Epilepsy Behav 62:104-114. https://doi.org/10.1016/j.yebeh.2016.06.014

Mcduff D, Kodra E, El Kaliouby R, Lafrance M (2017). A Large-Scale Analysis of Sex Differences in Facial Expressions. https://doi.org/ 10.1371/journal.pone. 0173942

Mégevand P, Groppe DM, Goldfinger MS et al (2014) Seeing scenes: topographic visual hallucinations evoked by direct electrical stimulation of the parahippocampal place area. J Neurosci 34:53995405. https://doi.org/10.1523/JNEUROSCI.5202-13.2014

Mitterschiffthaler MT, Fu CHY, Dalton JA et al (2007) A functional MRI study of happy and sad affective states induced by classical music. Hum Brain Mapp 28:1150-1162. https://doi.org/10.1002/ hbm. 20337

Morcuende S, Delgado-Garcia J-M, Ugolini G (2002) Neuronal premotor networks involved in eyelid responses: retrograde transneuronal tracing with rabies virus from the orbicularis oculi muscle in the rat. J Neurosci 22:8808-8818

Morecraft RJ, Louie JL, Herrick JL, Stilwell-Morecraft KS (2001) Cortical innervation of the facial nucleus in the non-human primate: a new interpretation of the effects of stroke and related subtotal brain trauma on the muscles of facial expression. Brain 124:176-208

Morecraft RJ, Cipolloni PB, Stilwell-Morecraft KS et al (2004) Cytoarchitecture and cortical connections of the posterior cingulate and adjacent somatosensory fields in the rhesus monkey. J Comp Neurol 469:37-69. https://doi.org/10.1002/cne.10980

Muakkassa KF, Strick PL (1979) Frontal lobe inputs to primate motor cortex: evidence for four somatotopically organized "premotor" areas. Brain Res 177:176-182. https://doi.org/10.1016/00068993(79)90928-4

Norman-Haignere SV, McCarthy G, Chun MM, Turk-Browne NB (2012) Category-selective background connectivity in ventral visual cortex. Cereb Cortex 22:391-402. https://doi.org/10.1093/ cercor/bhr118

Parvizi J, Jacques C, Foster BL et al (2012) Electrical stimulation of human fusiform face-selective regions distorts face perception. J Neurosci 32:14915-14920. https://doi.org/10.1523/JNEUROSCI. 2609-12.2012

Peltola ME, Trébuchon A, Lagarde S et al (2020) Anatomoelectroclinical features of SEEG-confirmed pure insular-onset epilepsy. Epilepsy Behav. https://doi.org/10.1016/j.yebeh.2020.106964

Pestana EM, Gupta A (2007) Ipsilateral blinking seizures during left fronto-temporal ictal pattern on scalp EEG. Epileptic Disord 9:449-452. https://doi.org/10.1684/epd.2007.0130

Peters J, Daum I, Gizewski E et al (2009) Associations evoked during memory encoding recruit the context-network. Hippocampus 19:141-151. https://doi.org/10.1002/hipo.20490 
Ponder E, Kennedy WP (1927) On the act of blinking. J Exp Physiol 18:89-110. https://doi.org/10.1113/expphysiol.1927.sp000433

Raslan A, Volk GF, Möller M et al (2017) High variability of facial muscle innervation by facial nerve branches: a prospective electrostimulation study. Laryngoscope 127:1288-1295. https://doi. org/10.1002/lary.26349

Schulte-Rüther M, Markowitsch HJ, Shah NJ et al (2008) Gender differences in brain networks supporting empathy. Neuroimage 42:393-403. https://doi.org/10.1016/j.neuroimage.2008.04.180

Sindou M, Fobe JL, Berthie E, Vial C (1994) Facial motor responses evoked by direct electrical stimulation of the trigeminal root. Localizing value for radiofrequency thermorhizotomy. Acta Neurochir (wien) 128:57-67. https://doi.org/10.1007/BF01400654

Smit AE (2009) Blinking and the Brain: Pathways and Pathology

Smith A, Henson R, Dolan R, Rugg M (2004) fMRI correlates of the episodic retrieval of emotional contexts. Neuroimage 22:868-878. https://doi.org/10.1016/J.NEUROIMAGE.2004.01.049

Tendolkar I, Arnold J, Petersson KM et al (2008) Contributions of the medial temporal lobe to declarative memory retrieval:
Manipulating the amount of contextual retrieval. Learn Mem 15:611-617. https://doi.org/10.1101/lm.916708

Urban PP, Wicht S, Marx J et al (1998) Isolated voluntary facial paresis due to pontine ischemia. Neurology 50:1859-1862

Wang H, McGonigal A, Zhang K et al (2020) Semiologic subgroups of insulo-opercular seizures based on connectional architecture atlas. Epilepsia 61:984-994. https://doi.org/10.1111/epi.16501

Yoon HW, Chung JY, Song MS, Park H (2005) Neural correlates of eye blinking; improved by simultaneous fMRI and EOG measurement. Neurosci Lett 381:26-30. https://doi.org/10.1016/j.neulet. 2005.01.077

Publisher's Note Springer Nature remains neutral with regard to jurisdictional claims in published maps and institutional affiliations. 